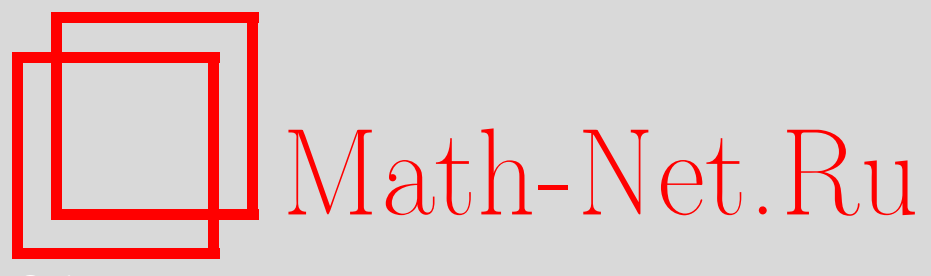

М. С. Вязовская, Н. С. Пупашенко, О нормирующем множителе обобщенного ядра Джексона, Матем. заметки, 2006, том 80, выпуск 1, 20-28

DOI: https://doi.org/10.4213/mzm2775

Использование Общероссийского математического портала Math-Net.Ru подразумевает, что вы прочитали и согласны с пользовательским соглашением http://www .mathnet.ru/rus/agreement

Параметры загрузки:

IP : 54.197 .217 .227

26 апреля 2023 г., 13:51:09

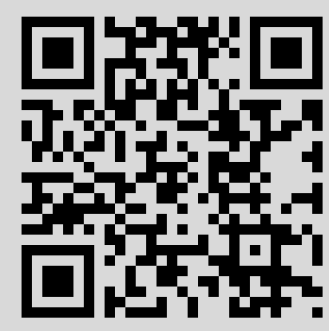




\section{О НОРМИРУЮЩЕМ МНОЖИТЕЛЕ \\ ОБОБЩЕННОГО ЯДРА ДЖЕКСОНА}

\section{М. С. Вязовская, Н. С. Пупашенко}

Рассматривается вопрос о значении нормирующего множителя

$$
\gamma_{n, k}=\frac{1}{\pi} \int_{-\pi}^{\pi}\left(\frac{\sin \frac{n t}{2}}{\sin \frac{t}{2}}\right)^{2 k} d t
$$

для обобщенного ядра Джексона $J_{n, k}(t)$. Получены явная формула

$$
\gamma_{n, k}=2 \sum_{p=0}^{\left[k-\frac{k}{n}\right]}(-1)^{p}\left(\begin{array}{c}
2 k \\
p
\end{array}\right)\left(\begin{array}{c}
k(n+1)-n p-1 \\
k(n-1)-n p
\end{array}\right)
$$

и представление

$$
\gamma_{n, k}=\sqrt{\frac{24}{\pi}} \cdot \frac{(n-1)^{2 k-1}}{\sqrt{2 k-1}}\left[1-\frac{1}{8} \cdot \frac{1}{2 k-1}+\omega(n, k)\right],
$$

где

$$
|\omega(n, k)|<\frac{4}{(2 k-1) \sqrt{\ln (2 k-1)}}+\sqrt{12 \pi} \cdot \frac{k^{\frac{3}{2}}}{n-1}\left(1+\frac{1}{n-1}\right)^{2 k-2} .
$$

Библиография: 9 названий.

1. Введение. В теории приближений хорошо известно обобщенное ядро Джексона (см. монографии по теории аппроксимации [1]-[7] и др.)

$$
J_{n, k}(t)=\frac{1}{\gamma_{n, k}}\left(\frac{\sin n t / 2}{\sin t / 2}\right)^{2 k}
$$

в котором

$$
\gamma_{n, k}=\frac{1}{\pi} \int_{-\pi}^{\pi}\left(\frac{\sin \frac{n t}{2}}{\sin \frac{t}{2}}\right)^{2 k} d t
$$

Хорошо известно (см. [8]), что

$$
c_{1}(k) n^{2 k-1} \leqslant \gamma_{n, k} \leqslant c_{2}(k) n^{2 k-1},
$$

где $c_{1}(k), c_{2}(k)$ - постоянные, зависящие от $k$.

Ввиду важности ядра $J_{n, k}$ для приложений возникает вопрос о более точной оценке значения $\gamma_{n, k}$ для различных $n$ и $k$.

В данной работе в пункте 2 доказана

(C) М. С. ВЯзовСКАЯ, Н. С. ПУПАшЕНко, 2006 
ТЕоремА 1. Значение $\gamma_{n, k}$ равно удвоенному коэфбициенту при $x^{k(n-1)}$ в многочлене $\left(1+x+\cdots+x^{n-1}\right)^{2 k}$.

С помощью теоремы 1 получено равенство

$$
\gamma_{n, k}=2 \sum_{p=0}^{\left[k-\frac{k}{n}\right]}(-1)^{p}\left(\begin{array}{c}
2 k \\
p
\end{array}\right)\left(\begin{array}{c}
k(n+1)-n p-1 \\
k(n-1)-n p
\end{array}\right) .
$$

В пункте 3 доказана следующая оценка для $\gamma_{n, k}$.

Теорема 2. Для всех $n, k \geqslant 2$ имеет место равенство

$$
\gamma_{n, k}=\sqrt{\frac{24}{\pi}} \cdot \frac{(n-1)^{2 k-1}}{\sqrt{2 k-1}}\left[1-\frac{1}{8} \cdot \frac{1}{2 k-1}+\omega(n, k)\right],
$$

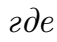

$$
|\omega(n, k)|<\frac{4}{(2 k-1) \sqrt{\ln (2 k-1)}}+\sqrt{12 \pi} \cdot \frac{k^{\frac{3}{2}}}{n-1}\left(1+\frac{1}{n-1}\right)^{2 k-2} .
$$

2. Явная формула для $\gamma_{n, k}$. Всюду далее $\sharp A$ означает количество элементов множества $A$. Обозначим

$$
\zeta_{i_{1}, \ldots, i_{m}}:=\left\{\xi=\left(\xi_{1}, \ldots, \xi_{m}\right) \in\{-1,1\}^{m} \mid \sum_{j=1}^{m} \xi_{j} i_{j}=0\right\} .
$$

Нам понадобится следующая лемма.

Лемма 1. Имеет место равенство

$$
\gamma_{n, k}=2 \sum_{m=0}^{k}\left(\begin{array}{c}
k \\
m
\end{array}\right) n^{k-m} \sum_{i_{1}, \ldots, i_{m}=1}^{n-1} \prod_{j=1}^{m}\left(n-i_{j}\right) \sharp \zeta_{i_{1}, \ldots, i_{m}} .
$$

ДокАЗАТЕЛЬСтво. Имеем

$$
\begin{aligned}
\gamma_{n, k} & =\frac{1}{\pi} \int_{-\pi}^{\pi}\left(\frac{\sin \frac{n t}{2}}{\sin \frac{t}{2}}\right)^{2 k} d t=\frac{1}{\pi} \int_{-\pi}^{\pi}\left(n+2 \sum_{p=1}^{n-1}(n-p) \cos p t\right)^{k} d t \\
& =\frac{1}{\pi} \int_{-\pi}^{\pi} \sum_{m=0}^{k}\left(\begin{array}{c}
k \\
m
\end{array}\right) n^{k-m} 2^{m} \sum_{i_{1}, \ldots, i_{m}=1}^{n-1} \prod_{j=1}^{m}\left(n-i_{j}\right) \cos i_{j} t d t \\
& =2 \sum_{m=0}^{k}\left(\begin{array}{c}
k \\
m
\end{array}\right) n^{k-m} \sum_{i_{1}, \ldots, i_{m}=1}^{n-1} \prod_{j=1}^{m}\left(n-i_{j}\right) \cdot \frac{2^{m-1}}{\pi} \int_{-\pi}^{\pi} \prod_{j=1}^{m} \cos i_{j} t d t .
\end{aligned}
$$

С помощью индукции по $m$ находим

$$
\int_{-\pi}^{\pi} \prod_{j=1}^{m} \cos i_{j} t d t=\frac{1}{2^{m}} \sum_{\xi \in\{-1,1\}^{m}} \int_{-\pi}^{\pi} \cos \left(\sum_{j=1}^{m} \xi_{j} i_{j}\right) t d t .
$$

Поэтому из равенства

$$
\int_{-\pi}^{\pi} \cos a x d x= \begin{cases}0, & \text { если } a \in \mathbb{Z} \backslash\{0\} \\ 2 \pi, & \text { если } a=0\end{cases}
$$


следует, что

$$
\frac{2^{m-1}}{\pi} \int_{-\pi}^{\pi} \prod_{j=1}^{m} \cos i_{j} t d t=\sharp \zeta_{i_{1}, \ldots, i_{m}} .
$$

Лемма доказана.

ДоКАЗАТЕЛЬСТво тЕОРЕМЫ 1 . Заметим, что $\sharp \zeta_{i_{1}, \ldots, i_{m}}$ равно свободному члену в выражении

$$
\left(x^{i_{1}}+x^{-i_{1}}\right) \ldots\left(x^{i_{m}}+x^{-i_{m}}\right) .
$$

Тогда из леммы 1 вытекает, что $\frac{1}{2} \gamma_{n, k}$ равно свободному члену в выражении

$$
\sum_{m=0}^{k}\left(\begin{array}{c}
k \\
m
\end{array}\right) n^{k-m} \sum_{i_{1}, \ldots, i_{m}=1}^{n-1} \prod_{j=1}^{m}\left(n-i_{j}\right)\left(x^{i_{j}}+x^{-i_{j}}\right):=B .
$$

В свою очередь,

$$
\begin{aligned}
B & =\sum_{m=0}^{k}\left(\begin{array}{c}
k \\
m
\end{array}\right) n^{k-m}\left(\sum_{i=1}^{n-1}(n-i)\left(x^{i}+x^{-i}\right)\right)^{m} \\
& =\left(n+\left(\sum_{i=1}^{n-1}(n-i)\left(x^{i}+x^{-i}\right)\right)\right)^{k} .
\end{aligned}
$$

Теперь требуемый результат следует из равенств

$$
\begin{aligned}
n+\left(\sum_{i=1}^{n-1}(n-i)\left(x^{i}+x^{-i}\right)\right) & =\sum_{i=0}^{n-1} \sum_{p=-i}^{i} x^{p} \\
=\sum_{i=0}^{n-1} x^{-i} \frac{1-x^{2 i+1}}{1-x} & =\sum_{i=0}^{n-1} \frac{x^{-i}}{1-x}-\sum_{i=0}^{n-1} \frac{x^{i+1}}{1-x} \\
= & \frac{1}{x^{n-1}}\left(\frac{1-x^{n}}{1-x}\right)^{2}=\left(1+x+\cdots+x^{n-1}\right)^{2} \frac{1}{x^{n-1}} .
\end{aligned}
$$

Теорема доказана.

СледСтвиЕ 1. Имеет место равенство

$$
\gamma_{n, k}=2 \sum_{p=0}^{\left[k-\frac{k}{n}\right]}(-1)^{p}\left(\begin{array}{c}
2 k \\
p
\end{array}\right)\left(\begin{array}{c}
k(n+1)-n p-1 \\
k(n-1)-n p
\end{array}\right) .
$$

ДокАЗАТЕЛЬСтвО. Легко видеть, что

$$
\left(\sum_{m=0}^{\infty} x^{m}\right)^{2 k}=\sum_{m=0}^{\infty}\left(\begin{array}{c}
2 k+m-1 \\
m
\end{array}\right) x^{m}, \quad x \in(-1,1) .
$$

Поэтому $\forall x \in(-1 ; 1)$ имеем

$$
\begin{aligned}
\left(1+x+\cdots+x^{n-1}\right)^{2 k} & =\left(\frac{1-x^{n}}{1-x}\right)^{2 k}=\left(\sum_{m=0}^{\infty} x^{m}\right)^{2 k}\left(1-x^{n}\right)^{2 k} \\
& =\sum_{i=0}^{\infty}\left(\begin{array}{c}
2 k+i-1 \\
i
\end{array}\right) x^{i} \sum_{p=0}^{2 k}\left(\begin{array}{c}
2 k \\
p
\end{array}\right)\left(-x^{n}\right)^{p}
\end{aligned}
$$


Теперь (2) следует из теоремы 1.

Приведем значения $\gamma_{n, k}$ для малых $k$ :

\begin{tabular}{|c|c|}
\hline$k$ & $\gamma_{n, k}$ \\
\hline$k=1$ & $2 n$ \\
\hline$k=2$ & $\frac{4}{3} n^{3}+\frac{2}{3} n$ \\
\hline$k=3$ & $\frac{11}{10} n^{5}+\frac{1}{2} n^{3}+\frac{2}{5} n$ \\
\hline$k=4$ & $\frac{302}{315} n^{7}+\frac{4}{9} n^{5}+\frac{14}{45} n^{3}+\frac{2}{7} n$ \\
\hline$k=5$ & $\frac{15619}{18144} n^{9}+\frac{175}{432} n^{7}+\frac{247}{864} n^{5}+\frac{1025}{4536} n^{3}+\frac{2}{9} n$ \\
\hline$k=6$ & $\frac{655177}{831600} n^{11}+\frac{809}{2160} n^{9}+\frac{961}{3600} n^{7}+\frac{3197}{15120} n^{5}+\frac{479}{2700} n^{3}+\frac{2}{11} n$ \\
\hline
\end{tabular}

СлЕДСТвиЕ 2. Справедливо равенство

$$
\begin{aligned}
\frac{\gamma_{n, k}}{2} & =\sharp\left\{\xi \in\{0, \ldots, n-1\}^{2 k} \mid \sum_{j=1}^{2 k} \xi_{j}=k(n-1)\right\} \\
& =\sharp\left\{\xi \in\left\{0, \frac{1}{n-1}, \frac{2}{n-1}, \ldots, 1\right\}^{2 k} \mid \sum_{j=1}^{2 k} \xi_{j}=k\right\} .
\end{aligned}
$$

3. Доказательство теоремы 2. Нам понадобятся следующие вспомогательные факты. Обозначим

$$
\varphi(z)=\frac{\sin z / 2}{z / 2}
$$

Имеет место следующее утверждение

ЛЕмма 2. При $|z| \leqslant 3$ имеем

$$
\ln \varphi(z)=S_{2} z^{2}+S_{4} z^{4}+\beta(z) z^{6},
$$

где $S_{2}=-1 / 24, S_{4}=-1 / 2880, a|\beta(z)| \leqslant 0.00011$.

ДокАЗАТЕЛЬСтво. Используя разложение $\ln \varphi(z)$ в ряд Тейлора в окрестности нуля с остаточным членом в форме Лагранжа, получаем

$$
\begin{aligned}
\ln \left(\frac{\sin \frac{z}{2}}{\frac{z}{2}}\right) & =\left(\frac{\sin \frac{z}{2}}{\frac{z}{2}}-1\right)-\frac{1}{2}\left(\frac{\sin \frac{z}{2}}{\frac{z}{2}}-1\right)^{2}-\frac{1}{3}\left(\frac{\sin \frac{z}{2}}{\frac{z}{2}}-1\right)^{3} \cdot \alpha \\
& =-\frac{z^{2}}{4 \cdot 3 !}+\frac{z^{4}}{16 \cdot 5 !}-\frac{z^{6} \cdot \theta_{1}}{64 \cdot 7 !}-\frac{1}{2}\left(-\frac{z^{2}}{4 \cdot 3 !}+\frac{z^{4} \cdot \theta_{2}}{16 \cdot 5 !}\right)^{2}+\frac{\alpha}{3}\left(\frac{z^{2} \cdot \theta_{3}}{4 \cdot 3 !}\right)^{3} \\
& =-\frac{z^{2}}{24}-\frac{z^{4}}{2880}+z^{6}\left(-\frac{\theta_{1}}{64 \cdot 7 !}+\frac{\theta_{2}}{64 \cdot 3 ! \cdot 5 !}+\frac{\theta_{3}^{3} \cdot \alpha}{3 \cdot 24^{3}}\right)+\frac{z^{8} \cdot \theta_{2}^{2}}{2 \cdot(16 \cdot 5 !)^{2}} \\
& =-\frac{z^{2}}{24}-\frac{z^{4}}{2880}+z^{6}\left(-\frac{\theta_{1}}{64 \cdot 7 !}+\frac{\theta_{2}}{64 \cdot 3 ! \cdot 5 !}+\frac{\theta_{3}^{3} \cdot \alpha}{3 \cdot 24^{3}}+\frac{z^{2} \cdot \theta_{2}^{2}}{2 \cdot(16 \cdot 5 !)^{2}}\right),
\end{aligned}
$$

где $\theta_{i} \in[-1 ; 1], i=1, \ldots, 3$, а $\alpha=z_{0}^{-3}$, где $z_{0} \in[\sin (z / 2) /(z / 2) ; 1]$. Легко видеть, что из $|z| \leqslant 3$ следует, что $\sin (z / 2) /(z / 2)>0.66$, откуда $\alpha<3.48$. Поэтому

$$
\left|-\frac{\theta_{1}}{64 \cdot 7 !}+\frac{\theta_{2}}{64 \cdot 3 ! \cdot 5 !}+\frac{\theta_{3}^{3} \cdot \alpha}{3 \cdot 24^{3}}+\frac{z^{2} \cdot \theta_{2}^{2}}{2 \cdot(16 \cdot 5 !)^{2}}\right|<0.00011,
$$


что и требовалось доказать.

Зафиксируем $p \in \mathbb{N}$ и обозначим

$$
I:=\int_{-\infty}^{\infty}\left|\left(\varphi\left(\frac{z}{\sqrt{p}}\right)\right)^{p}-e^{S_{2} z^{2}}\left(1+\frac{S_{4} z^{4}}{p}\right)\right| d z .
$$

Лемма 3. Имеет место оценка

$$
I \leqslant \frac{22}{p \sqrt{\ln p}} .
$$

ДокАЗАТЕЛЬСтво. Очевидно, что для любого $K>0$

$$
\begin{aligned}
I \leqslant \int_{|z|>\sqrt{K \ln p}}\left|\varphi\left(\frac{z}{\sqrt{p}}\right)\right|^{p} d z+\int_{|z|>\sqrt{K \ln p}}\left|e^{S_{2} z^{2}}\left(1+\frac{S_{4} z^{4}}{p}\right)\right| d z \\
\quad+\int_{|z| \leqslant \sqrt{K \ln p}}\left|\left(\varphi\left(\frac{z}{\sqrt{p}}\right)\right)^{p}-e^{S_{2} z^{2}}\left(1+\frac{S_{4} z^{4}}{p}\right)\right| d z=: I_{A}+I_{B}+I_{C} .
\end{aligned}
$$

Положим $K=24$ и оценим каждый из полученных интегралов:

$$
\begin{aligned}
I_{A} & =\sqrt{p} \cdot \int_{|z|>\sqrt{\frac{24 \ln p}{p}}}|\varphi(z)|^{p} d z=\sqrt{p} \cdot \int_{|z|>\sqrt{\frac{24 \ln p}{p}}}\left|\frac{\sin z / 2}{z / 2}\right|^{p} d z \\
& \leqslant 2\left(\sqrt{p} \int_{\sqrt{\frac{24 \ln p}{p}}}^{4}\left(e^{-\frac{z^{2}}{24}}\right)^{p} d z+\sqrt{p} \int_{4}^{\infty}\left(\frac{2}{z}\right)^{p} d z\right) \\
& \leqslant 2 \int_{\sqrt{24 \ln p}}^{\infty} e^{-\frac{y^{2}}{24}} d y+\frac{2 \sqrt{p}}{(p-1) 2^{p-1}} \\
& \leqslant 2 \int_{\sqrt{24 \ln p}}^{\infty} e^{-\frac{y \cdot \sqrt{24 \ln p}}{24}} d y+\frac{2 \sqrt{p}}{(p-1) 2^{p-1}} \leqslant \frac{3}{p \sqrt{\ln p}}, \\
I_{B} & =\int_{|z|>\sqrt{K \ln p}} e^{-\frac{1}{24} z^{2}} d z+\frac{2 S_{4}}{p}\left(\int_{\sqrt{K \ln p}}^{\infty} z^{4} e^{-\frac{1}{24} z^{2}} d z\right) \\
& \leqslant \int_{\sqrt{K \ln p}}^{\infty} e^{-\frac{1}{24} z \sqrt{24 \ln p}} d z\left(2+\frac{6}{20 p}\right)+\frac{\sqrt{24}(\ln p)^{\frac{3}{2}}}{5 p^{2}}+\frac{3 \cdot \sqrt{24}(\ln p)^{\frac{1}{2}}}{10 p^{2}} \\
& \leqslant \frac{1}{p \sqrt{\ln p}}\left(\frac{1}{\sqrt{6}}+\frac{2 \sqrt{6}(\ln p)^{2}}{p}+\frac{3 \sqrt{6} \ln p}{p}+\frac{3}{20 \sqrt{6} p}\right) \leqslant \frac{2}{p \sqrt{\ln p}} .
\end{aligned}
$$

Для оценки $I_{C}$ заметим, что из леммы 2 следует

$$
\begin{aligned}
\left(\varphi\left(\frac{z}{\sqrt{p}}\right)\right)^{p} & =\exp \left\{S_{2} z^{2}+\frac{z^{4}}{p} S_{4}+\frac{z^{6}}{p^{2}} \beta\left(\frac{z}{\sqrt{p}}\right)\right\} \\
& =\exp \left\{S_{2} z^{2}\right\} \exp \left\{\frac{z^{4}}{p} S_{4}+\frac{z^{6}}{p^{2}} \beta\left(\frac{z}{\sqrt{p}}\right)\right\} \\
& =\exp \left\{S_{2} z^{2}\right\}\left(\frac{z^{4}}{p} S_{4}+\frac{z^{6}}{p^{2}} \beta\left(\frac{z}{\sqrt{p}}\right)+\left(\frac{z^{4}}{p} S_{4}+\frac{z^{6}}{p^{2}} \beta\left(\frac{z}{\sqrt{p}}\right)\right)^{2} \gamma(z)\right)
\end{aligned}
$$


где $\gamma(z)<1 / 2$. Теперь, так как $\sqrt{24 \ln p / p}<3$, из леммы 2 окончательно получаем

$$
\begin{aligned}
I_{C} & =\int_{|z| \leqslant \sqrt{24 \ln p}}\left(\frac{z^{6}}{p^{2}} \beta\left(\frac{z}{\sqrt{p}}\right)+\left(\frac{z^{4}}{p} S_{4}+\frac{z^{6}}{p^{2}} \beta\left(\frac{z}{\sqrt{p}}\right)\right)^{2} \gamma(z)\right) \\
& \leqslant 2.2 \frac{(\ln p)^{\frac{7}{2}}}{p^{2}}+0.03 \frac{(\ln p)^{\frac{9}{2}}}{p^{2}}+0.3 \frac{(\ln p)^{\frac{11}{2}}}{p^{3}}+0.9 \frac{(\ln p)^{\frac{13}{2}}}{p^{4}} \\
& \leqslant 2.2 \frac{(\ln p)^{\frac{7}{2}}}{p^{2}}+0.3 \frac{(\ln p)^{\frac{9}{2}}}{p^{2}} .
\end{aligned}
$$

Легко убедиться, что для всех $p \in \mathbb{N}$ выполняется неравенство

$$
\frac{2.2(\ln p)^{\frac{7}{2}}}{p^{2}}+\frac{0.3(\ln p)^{\frac{9}{2}}}{p^{2}} \leqslant \frac{17}{p \sqrt{\ln p}}
$$

откуда и следует утверждение леммы.

Пусть

$$
M=\left\{x=\left(x_{1}, \ldots, x_{2 k-1}\right) \in[0 ; 1]^{2 k-1} \mid \sum_{j=1}^{2 k-1} x_{j} \in[k-1 ; k]\right\},
$$

a $V(M)$ - объем тела $M$. Имеет место

Лемма 4. Справедлива оценка

$$
\left|(n-1)^{2 k-1} V(M)-\frac{\gamma_{n, k}}{2}\right| \leqslant 6 k \cdot n^{2 k-2} .
$$

ДокАзАТЕЛЬСтво. Для удобства введем следующие обозначения:

$$
\begin{aligned}
L & =\left\{\left(\frac{l_{1}}{n-1}, \ldots, \frac{l_{2 k-1}}{n-1}\right) \mid l_{j}=0, \ldots, n-1\right\}-\text { решетка в кубе }[0 ; 1]^{2 k-1}, \\
L^{*} & =\left\{\left(\frac{l_{1}}{n-1}, \ldots, \frac{l_{2 k}}{n-1}\right) \mid l_{j}=0, \ldots, n-1\right\}-\text { решетка в кубе }[0 ; 1]^{2 k} .
\end{aligned}
$$

Тогда

$$
\begin{aligned}
\frac{\gamma_{n, k}}{2} & =\sharp\left\{\xi \in L^{*} \mid \sum_{j=1}^{2 k} \xi_{j}=k\right\} \\
& =\sharp\left\{\xi \in L^{*} \mid \sum_{j=1}^{2 k-1} \xi_{j} \in[k-1 ; k], \quad \xi_{2 k}=k-\sum_{j=1}^{2 k-1} \xi_{j}\right\} \\
& =\sharp\left\{\xi \in L \mid k-1 \leqslant \sum_{j=1}^{2 k} \xi_{j} \leqslant k\right\}=: N,
\end{aligned}
$$

т.е. $\gamma_{n, k} / 2=\sharp(L \cap M)$. Обозначим для $l \in L$

$$
B_{l}:=\prod_{j=1}^{2 k-1}\left[\frac{l_{j}}{n-1}-\frac{1}{2(n-1)} ; \frac{l_{j}}{n-1}+\frac{1}{2(n-1)}\right] .
$$


Для $x \in[0 ; 1]^{2 k-1}$ обозначим $\xi(x)$ ближайшую к $x$ точку решетки $L$. Тогда $\left|\xi_{i}(x)-x_{i}\right| \leqslant 1 /(2(n-1))$, откуда, при $x \in M$

$$
k-1-\frac{2 k-1}{2(n-1)} \leqslant \sum_{j=1}^{2 k-1} \xi_{j}(x) \leqslant k+\frac{2 k-1}{2(n-1)},
$$

а при $x \in[0 ; 1]^{2 k-1} \backslash M$

$$
k-\frac{2 k-1}{2(n-1)} \leqslant \sum_{j=1}^{2 k-1} \xi_{j}(x) \quad \text { или } \sum_{j=1}^{2 k-1} \xi_{j}(x) \leqslant k-1+\frac{2 k-1}{2(n-1)} .
$$

Тогда $[0 ; 1]^{2 k-1} \cap\left(\bigcup_{\xi \in N_{-}} B_{\xi}\right) \subset M \subset \bigcup_{\xi \in N_{+}} B_{\xi}$, где

$$
\begin{aligned}
& N_{+}=\left\{\xi \in L \mid k-1-\frac{2 k-1}{2(n-1)} \leqslant \sum_{j=1}^{2 k-1} \xi_{j} \leqslant k+\frac{2 k-1}{2(n-1)}\right\}, \\
& N_{-}=\left\{\xi \in L \mid k-1+\frac{2 k-1}{2(n-1)} \leqslant \sum_{j=1}^{2 k-1} \xi_{j} \leqslant k-\frac{2 k-1}{2(n-1)}\right\} .
\end{aligned}
$$

Зафиксировав первые $2 k-2$ координат, из неравенств выше получим, что

$$
\sharp N_{+} \leqslant \sharp N+n^{2 k-2}(2 k-1) \quad \text { и } \sharp N-n^{2 k-2}(2 k-1) \leqslant \sharp N_{-},
$$

откуда для объема $V(M)$ выполняются следующие оценки (для $N_{-}$необходимо отнять точки, лежащие на гранях куба $\left.[0 ; 1]^{2 k-1}\right)$ :

$$
\begin{aligned}
& V(M) \geqslant \frac{\sharp N_{-}}{(n-1)^{2 k-1}}-\frac{2(2 k-1)}{n-1} \geqslant \frac{\sharp N}{(n-1)^{2 k-1}}-\frac{6 k n^{2 k-2}}{(n-1)^{2 k-1}}, \\
& V(M) \leqslant \frac{1}{(n-1)^{2 k-1}} \sharp N_{+} \leqslant \frac{1}{(n-1)^{2 k-1}} \sharp N+\frac{(2 k-1) n^{2 k-2}}{(n-1)^{2 k-1}} .
\end{aligned}
$$

Так как $\gamma_{n, k} / 2=\sharp N$, из неравенств (3) и (4) получаем утверждение леммы.

Рассмотрим независимые случайные величины $\zeta_{j}$, равномерно распределенные на отрезке $[-1 / 2 ; 1 / 2]$. Заметим, что вероятность

$$
\mathrm{P}\left(\sum_{j=1}^{2 k-1} \zeta_{j} \in\left[-\frac{1}{2} ; \frac{1}{2}\right]\right)
$$

равна объему множества $M$. Для оценки полученной вероятности воспользуемся схемой доказательства граничной теоремы с уточнениями для случайных блужданий с непрерывным распределением (см. [9; теорема 5, с. 53]).

Характеристическая функция случайной величины $\zeta_{j}$ есть

$$
\varphi(z)=\frac{\sin z / 2}{z / 2} .
$$

Обозначим $f_{m}$ плотность распределения случайной величины

$$
\frac{1}{\sqrt{m}} \sum_{j=1}^{m} \zeta_{j}
$$


Лемма 5. Для всех $x$ имеет место следующая оченка:

$$
\left|f_{m}-\sqrt{\frac{6}{\pi}} e^{-6 x^{2}}\left[1+\frac{1}{m} Q_{2}(x)\right]\right| \leqslant \frac{1}{2 \pi} \cdot \frac{22}{m \sqrt{\ln m}},
$$

¿əe

$$
Q_{2}(x)=-\frac{3}{8}+\frac{27}{5} x^{2}-\frac{18}{5} x^{4}
$$

ДоказАтельство. Так как $\zeta_{j}$ независимые одинаково распределенные величины, характеристическая функция $\frac{1}{\sqrt{m}} \sum_{j=1}^{m} \zeta_{j}$ равна

$$
\varphi_{m}(z)=\left[\varphi\left(\frac{z}{\sqrt{m}}\right)\right]^{m}
$$

где $\varphi$ характеристическая функция величины $\zeta_{j}$. Поэтому

$$
f_{m}(x)=\frac{1}{2 \pi} \int_{-\infty}^{\infty} e^{-i z x}\left[\varphi\left(\frac{z}{\sqrt{m}}\right)\right]^{m} d z
$$

Отсюда в силу леммы 3

$$
\begin{aligned}
\left|f_{m}-\sqrt{\frac{6}{\pi}} e^{-6 x^{2}}\left[1+\frac{1}{m} Q_{2}(x)\right]\right| \\
\quad=\left|\frac{1}{2 \pi} \int_{-\infty}^{\infty}\left[\left(\varphi\left(\frac{z}{\sqrt{m}}\right)\right)^{m}-e^{S_{2} z^{2}}\left(1+\frac{1}{m} S_{4} z^{4}\right)\right] e^{-i z x} d z\right| \\
\quad \leqslant \frac{1}{2 \pi} \int_{-\infty}^{\infty}\left|\left(\varphi\left(\frac{z}{\sqrt{m}}\right)\right)^{m}-e^{S_{2} z^{2}}\left(1+\frac{1}{m} S_{4} z^{4}\right)\right| d z \leqslant \frac{1}{2 \pi} \cdot \frac{22}{m \sqrt{\ln m}} .
\end{aligned}
$$

Проинтегрировав по отрезку $[-1 /(2 \sqrt{2 k-1}) ; 1 /(2 \sqrt{2 k-1})]$ оценку, полученную для плотности $f_{m}$, находим

$$
\mathrm{P}\left(\sum_{j=1}^{2 k-1} \zeta_{j} \in\left[-\frac{1}{2} ; \frac{1}{2}\right]\right)=\sqrt{\frac{6}{\pi}}\left(\frac{1}{(2 k-1)^{\frac{1}{2}}}-\frac{1}{8(2 k-1)^{\frac{3}{2}}}+\delta(2 k-1)\right),
$$

где

$$
|\delta(p)| \leqslant \frac{4}{p^{\frac{3}{2}} \sqrt{\ln p}} .
$$

Отсюда из леммы 4 следует соотношение (1).

ЗАмЕчАнИЕ 1. Заметим, что оценка выполняется для всех $n, k$, однако представляет интерес лишь для $n \gg k$, скажем при $n>k^{3}$.

\section{СПИСОК ЦИТИРОВАННОЙ ЛИТЕРАТУРЫ}

[1] Н. И. Ахиезер, Лекции по теории апроксимации, Наука, М., 1965.

[2] R. A. DeVore, G. G. Lorentz, Constructive approximation, Springer-Verlag, Berlin, 1993.

[3] В.К. Дзядык, Введение в теорию равномерного приближения функиий полиномами, Наука, М., 1977.

[4] Н. П. Корнейчук, Экстремалъные задачи теории приближения, Наука, М., 1976. 
[5] С. М. Никольский, Приближение функций многих переменных и теоремы вложения, Наука, М., 1969.

[6] А.Ф. Тиман, Теория приближения функиий действительного переменного, Физматгиз, М., 1960.

[7] И. А. Шевчук, Приближение многочленами и следы непрерывных на отрезке функций, Наукова думка, Киев, 1992.

[8] С.Б. Стечкин, "О порядке наилучших приближений непрерывных функций”, Изв. АН. СССР. Сер. матем., 15:3 (1951), 219-242.

[9] А. В. Скороход, Н. П. Слободянюк, Предельные теоремы для случайных блужданий, Наукова думка, Киев, 1970.

\section{М. С. Вязовская}

Киевский национальный университет им. Тараса Шевченко E-mail: viazovska@univ.kiev.ua

\section{Н. С. Пупашенко}

Киевский национальный университет им. Тараса Шевченко

E-mail: kito@univ.kiev.ua
Поступила в редакцию 28.09.2005

Исправленный вариант 30.01.2006 